\title{
A cantilevered piezoelectric bi-stable composite concept for broadband energy harvesting
}

\author{
Andres F. Arrieta ${ }^{a}$, Tommaso Delpero ${ }^{a}$, Andrea Bergamini ${ }^{b}$, and Paolo Ermanni ${ }^{a}$ \\ ${ }^{a}$ Centre of Structure Technologies, ETH Zürich, Leonhardstrasse 27, Zürich, CH-8092 \\ Switzerland; \\ ${ }^{b}$ Empa, Mechanics for Modelling and Simulation, Überlandstrasse 129, CH-8600 Dübendorf
}

\begin{abstract}
Recently, the idea to exploit nonlinearity to achieve broadband energy harvesting has been introduced. Bi-stable systems have been used to realise broadband energy harvesting devices. Amongst these, harvesters constructed with bi-stable composites show great potential due to their rich dynamic behaviour. This paper studies a novel cantilevered configuration for a piezoelectric bi-stable composite device for broadband energy harvesting. The cantilevered configuration allows to exploit high strains developed close to the clamped root, further enhancing the harvesting characteristic of bi-stable composites. Furthermore, the desired broadband dynamics are obtained for lower input amplitudes when compared to previous designs constituting a significant improvement for energy harvesting applications. Several cross-well dynamic behaviours are obtained over a relatively wide range of frequencies with the proposed design. In addition, the performance of the developed concept is investigated using a switching shunt harvesting circuit suitable for conversion of broadband oscillations resulting from the cross-well dynamics exhibited by bi-stable composite laminates showing very good results.
\end{abstract}

Keywords: Energy harvesting, broadband, bi-stable composites, piezoelectricity, nonlinearity

\section{INTRODUCTION}

Vibration based energy harvesting has received extensive attention within the smart structures community during the last decade. ${ }^{1}$ Vibration-based energy harvesting devices mainly rely upon electromagnetic induction and piezoelectricity to convert ambient mechanical vibration from a host structure into usable forms. Some significant applications include powering wireless sensor systems, ${ }^{2}$ and autonomous battery recharging. ${ }^{3}$ Much of the research carried out on vibration-based energy harvesting has focused on devices tuned to operate close to a linear resonant frequency from the host structure. ${ }^{4}$ Although significant progress has been achieved in this direction, real harvesting devices would seldom operate in environments with such idealized conditions. Recently, the idea to exploit nonlinearity to achieve broadband energy harvesting has been introduced. ${ }^{5}$ Nonlinear systems have been shown to operate over a wide band of frequencies delivering high power. In particular, systems exhibiting bi-stability offer desired dynamic characteristics to achieve the objective of broadband high energy conversion. Amongst these, bi-stable composite plates show several advantages, including design flexibility for multiple resonance tuning owing to their two-dimensional nature, and reduced complexity as no external magnets are required to achieve bi-stability. ${ }^{6}$

In this paper, a novel cantilevered piezoelectric bi-stable composite plate concept is presented for broadband nonlinear energy harvesting. The cantilevered configuration offers enhanced response characteristics over previously proposed unconstrained bi-stable plates as larger strains close to the clamp end increase the effectiveness of the piezoelectric transducers. Furthermore, the current investigation couples the advantages of broadband conversion of bi-stable composites with a synchronised shunting harvesting on inductor circuit (SSHI) to enhance the harvesting capabilities of the employed piezoelectric transducers. The response of the harvesting device is investigated revealing several large amplitude nonlinear phenomena over a wide range of frequencies. Resistor sweeps are performed for representative dynamic regimes to obtain the optimal electrical load for each type of oscillations. The obtained experimental results for the proposed design show wide band frequency conversion maintaining large power output at relatively low base acceleration levels.

Further author information: (Send correspondence to A.F. Arrieta) A.F. Arrieta: E-mail: andresar@ethz.ch, Telephone: +41-(0)446322675

Active and Passive Smart Structures and Integrated Systems 2013, edited by Henry A. Sodano, Proc. of SPIE Vol. 8688, 86880G · C 2013 SPIE · CCC code: 0277-786X/13/\$18 doi: $10.1117 / 12.2010122$

Proc. of SPIE Vol. 8688 86880G-1 


\section{BI-STABLE COMPOSITES}

Bi-stable composites are a class of composites capable of attaining two statically stable shapes, ${ }^{7}$ which are schematically shown in red and green for the cantilevered configuration used in the present study in Fig. 1. Bi-stability appears due to the unsymmetrical lamination which induces thermal stresses during the cool down process from curing as a result of a mismatch between the thermal expansion coefficients of the fibres and the epoxy matrix. ${ }^{8}$ Forcing a large deflection on a bi-stable composite results in a sudden jump to the second stable state known as snap-through follows. This occurs when a given level of deflection is forced upon the

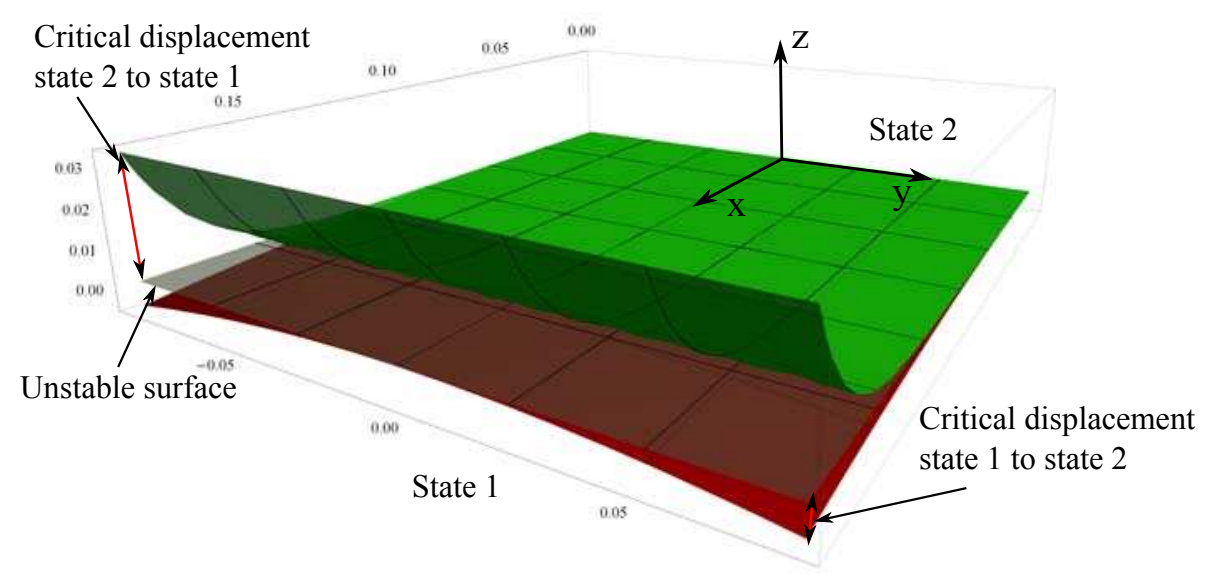

Figure 1. Stable states and critical displacement for a cantilevered bi-stable composite.

composite, known as critical displacement, after which snap-through ensues. ${ }^{9}$ When subjected to persistent external vibration causing deflections past the critical displacement of a bi-stable composite, constant snapthrough oscillations are triggered for which the configuration of the plate changes between the stable states. This type of dynamic behaviour described in literature as cross-well oscillations exhibit large amplitude response over a wide range of frequencies. ${ }^{9}$ Previous studies showed that cross-well dynamics are more easily triggered around the modal frequencies ${ }^{10}$ and nonlinear resonances ${ }^{11}$ of bi-stable composites. Hence, mechanical structures made of such composites can be designed such that many ranges of broadband dynamic response are obtained. These allows, for instance, for matching various resonances of a host structure, or clustering the linear and nonlinear resonances of the designed bi-stable composite around the range where the ambient vibration frequency is expected to be.

In view of the mentioned dynamic characteristics bi-stable composites are suitable for energy harvesting applications where the objective of broadband energy conversion is sought. In addition, bi-stable composites are easily integrated with piezoelectric transducers which facilitates the design of an energy harvesting component. Furthermore, the bi-stability in this type of composites is due to purely elastic phenomena, hence no additional parts such as bulky magnets are required to obtain the required dynamic behaviour resulting in a robust mechanical structure from which a reliable broadband energy harvester can be built from.

\subsection{Cantilevered configuration}

A cantilever configuration obtained by employing a symmetrical-unsymmetrical lay-up, ${ }^{12}$ as shown in as shown in Fig. 2, with two surface bonded piezoelectric transducers is used in the present investigation for three important reasons. First, this type of arrangement allows to exploit high strains developed close to the clamped root by the piezoelectric transducers. This constitutes an improvement over previously presented harvesting designs using bistable composites, while maintaining all the above mentioned advantages. Secondly, the cantilever configuration 


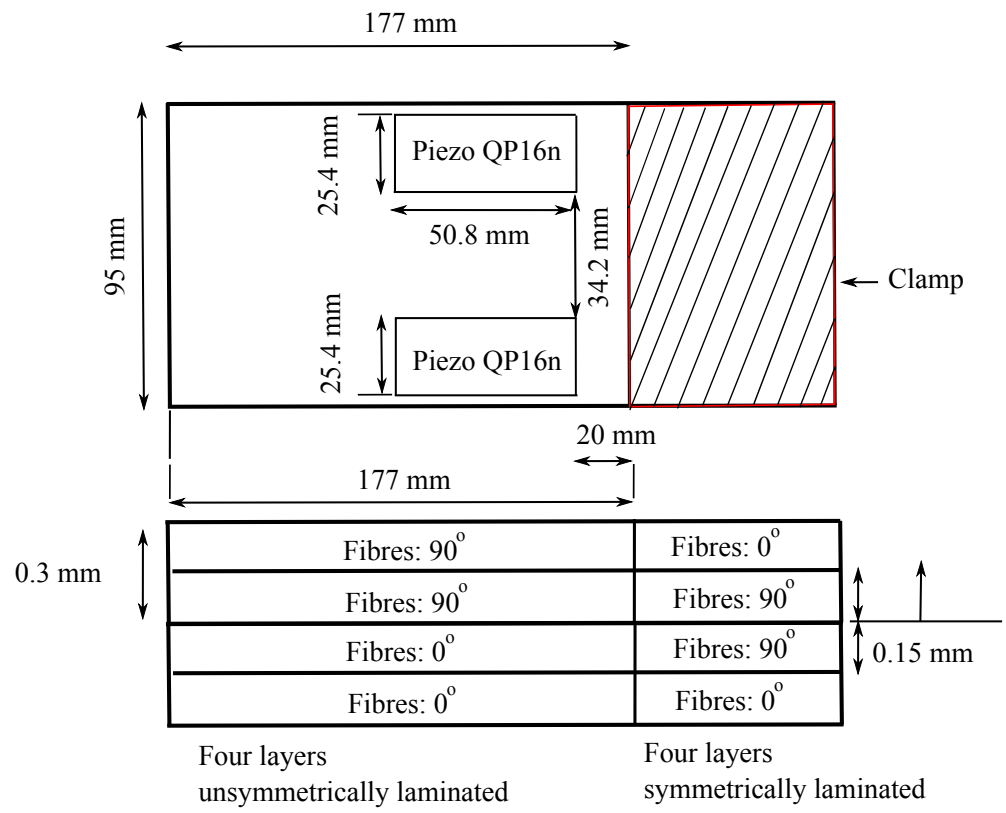

Figure 2. Symmetric-unsymmetric lay-up enabling cantilever configuration and positioning of piezoelectric transducers.

results in large displacements given the large distance from the root to the tip. This provides the current configuration with a significant advantage as the critical displacement to required to trigger span-through can be more easily reached, ${ }^{9}$ significantly reducing the vibration level needed for the appearance of the broadband crosswell oscillations sought after for efficient energy harvesting devices. Thirdly, having a cantilevered configuration allows for easier integration with the host structure. As a result of the above outlined reasons, the resulting cantilevered configuration allows for improved energy conversion in comparison to previous designs thanks to the piezoelectric transducers being positioned close to the clamped edge where the highest strains are developed.

\section{DYNAMIC RESPONSE}

A rectangular shape bi-stable composite made of carbon fibre-epoxy prepreg, type E022-T700 manufactured by $\mathrm{SLG},{ }^{13}$ with stacking sequence $\left[0_{2} / 90_{2}\right]$ is used for the experimental investigations. The cantilevered configuration described in section 2.1 is employed in which the symmetric part is used for clamping the bi-stable harvester to a base mounted on a shaker serving as the source of external vibration. A displacement signal is acquire with a laser sensor, and the acceleration level is measured with and accelerometer. The experimental set-up used in this investigation is shown in detail in Fig. 3.

\subsection{Low amplitude response}

In order to identify the vibration modes of the studied bi-stable harvester specimen a series of frequency response functions (FRF) for each stable state are measured using the base acceleration as input and the displacement of the composite laminate as output. The experimentally obtained modal frequencies for the first bending mode of stable state 1 and 2 are respectively $22.2 \mathrm{~Hz}$ and $16 \mathrm{~Hz}$. The obtained information for the low amplitude response of the studied rectangular bi-stable composite specimen is very useful for the subsequent study of the cross-well dynamics as these are triggered with the least amount of input around the first bending modes for bi-stable composites. ${ }^{10,11}$ Furthermore, designing the dimensions and lay-up of a particular bi-stable composites specimen allows to place in desired position the frequencies for the modes on each stable state using the model described in Ref. ${ }^{10}$ The cross-well dynamic behaviour is studied in the next section.

\subsection{Cross-well Dynamics}

To experimentally study the cross-well dynamic behaviour of the used specimen the same set-up as for the low amplitude response is used. In this case, a detailed study is carried out around the first bending mode of state 1. Frequency sweeps maintaining fixed the excitation levels are carried out with amplitude ranging from $0.1 \mathrm{~g}$ 


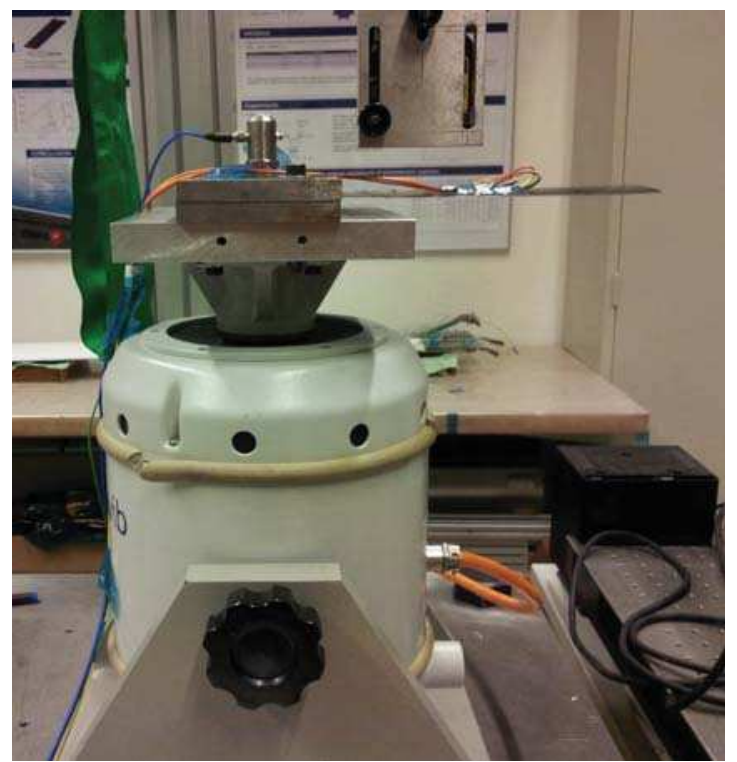

Figure 3. Experimental rig for the cantilever configuration of the piezoelectric bi-stable composite laminate.

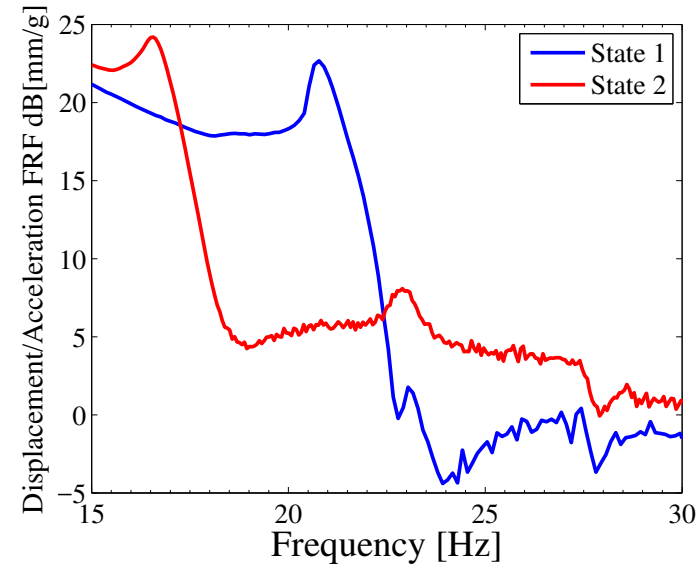

Figure 4. Experimental single-point out-of-plane displacement to base acceleration FRF of the bi-stable piezoelectric composite specimen in cantilevered configuration. Base acceleration level of $0.05 \mathrm{~g}$.

to $0.3 \mathrm{~g}$ in order to reveal the parameter space for which cross-well oscillations occur. It is found that cross-well oscillations are triggered with a minimum base acceleration level close to $0.15 \mathrm{~g}$ for the cantilevered configuration. At a level of $0.25 \mathrm{~g}$ a broad range of cross-well oscillations is obtained. In this range several cross-well dynamic behaviours are observed depending on the frequency at which the plate is excited, including chaotic oscillations and intermittency oscillations, as presented in Fig. 5.

The cross-well dynamics around the first bending mode of state 2 are not investigated in detail as its relative low frequency lies outside the range for which the used shaker could operate reliably. Nevertheless, indications of cross-well oscillations for similar acceleration levels as the ones seen around the first mode of state 1 could be observed. This further wides the possible range of cross-well dynamics for the proposed cantilevered piezoelectric bi-stable composite concept. Future work is planned to investigate this range with the use of a low frequency seismic shaker. 


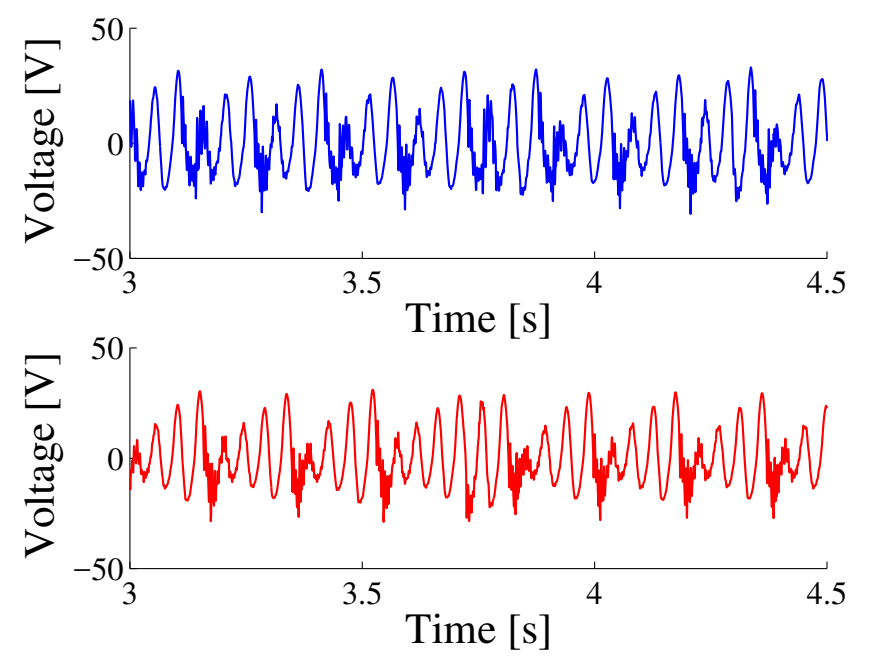

Figure 5. Experimental time series for open-circuit voltage output for constant base acceleration of $0.25 \mathrm{~g}$. a) fast chaotic oscillations at $19.5 \mathrm{~Hz}$. b) slow chaotic oscillations at $21.5 \mathrm{~Hz}$. Initial configuration state 1.

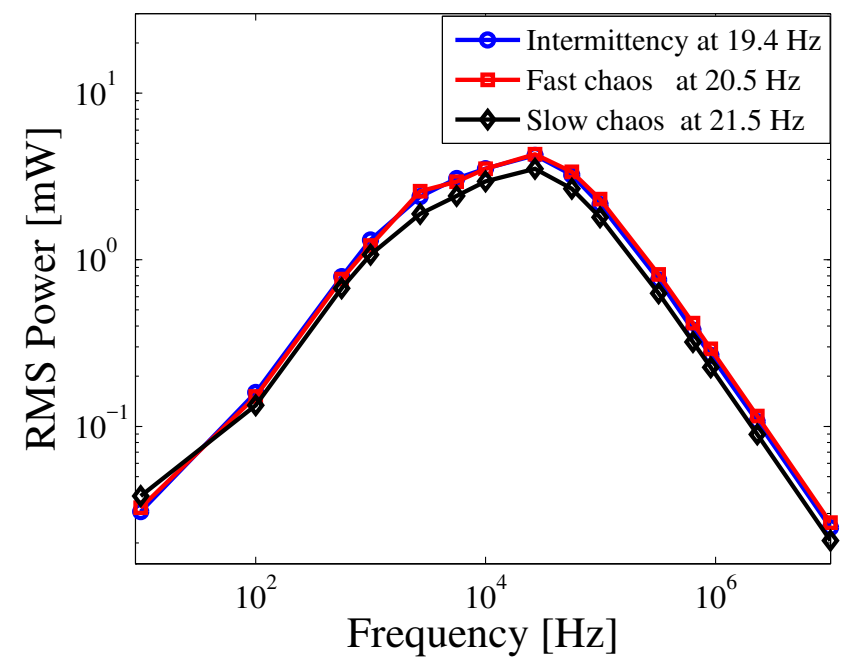

Figure 6. Experimental average power against load resistance for: intermittency oscillations at $19.5 \mathrm{~Hz}$, fast chaotic oscillations at $20.5 \mathrm{~Hz}$ and slow chaotic oscillations at $21.5 \mathrm{~Hz}$. Initial configuration state 1.

\section{ENERGY HARVESTING CHARACTERISTICS}

The proposed harvesting concept is tested by conducting a resistor sweep in order to identify the optimum resistance and maximum power achievable for the observed cross-well dynamic behaviours. To this end, typical time series for chaotic vibrations and intermittency oscillations measuring the voltage across a resistor load are obtained. Figure 6 presents the resulting power versus resistance curves from where it is observed that the optimum resistance value for each of the studied cross-well dynamic behaviours remains practically unchanged around a value of $27 \mathrm{k} \Omega$. This implies that a harvesting device made from such a concept will show robust harvesting characteristics over the different cross-well behaviours, highlighting the wide band conversion qualities of the current concept. Further, high RMS power is obtain at for the different types of nonlinear oscillations ranging between 3 to $6 \mathrm{~mW}$ for an acceleration input of $0.25 \mathrm{~g}$. These results show that the current configuration can deliver relatively high power even for an low acceleration level. Previously, high accelerations in excess of $1.5 \mathrm{~g}$ were required to obtain broadband oscillations for similar bi-stable composite concepts. ${ }^{14}$ 


\subsection{Broadband behaviour}

To assess the broadband energy harvesting characteristics of the current concept, the voltage and power conversion as a function of the frequency are investigated. Frequency sweeps for the converted voltage are obtained for a low amplitude excitation, which is regarded as close to the linear behaviour of the system. Assuming the hypothesis is right, the obtained low amplitude data can be scaled up by a factor matching a chosen input level for which the system behaves nonlinearly. This allows for a rough comparison between the actual nonlinear response of the proposed harvester to a hypothetical one given by a linear system. In particular, it provides an indication of the range for which large power can be converted from the ambient vibrations by studying the drop in converted voltage as the forcing frequency moves away from the linear resonance. As can be observed in the red dots Fig. 7, the range of frequencies for which the voltage is larger than $50 \%$ of the peak voltage is centered around the first resonance from $20.6 \mathrm{~Hz}$ to 22.4 , resultin in a range of $1.6 \mathrm{~Hz}$.

The nonlinear behaviour is studied by conducting the same type of frequency sweeps for a base acceleration input of $0.25 \mathrm{~g}$, which yields a significant range of cross-well oscillations as mentioned in section 3.2. Frequency response diagrams, similar to bifurcation diagrams, for which the amplitude of consecutive forcing frequencies are sampled and plotted as a function of the frequency are used to study the nonlinear response (for details see Ref. $\left.{ }^{15}\right)$. In this type of diagrams a single dot for a forcing frequency, levels indicates a linear behaviour, whereas several dots for a forcing frequency is a sign of nonlinear oscillations. In particular, for chaotic oscillations the amplitude of the response does not repeat periodically, hence a cloud of points for each forcing frequency is plotted in the frequency response diagrams. In blue dots Fig. 7 shows that, thanks to the bi-stability resulting in cross-well oscillations, the real nonlinear response shows a range of $3.4 \mathrm{~Hz}$, from 18.5 to $22.2 \mathrm{~Hz}$ for oscillations starting in state 1 . Although the peak value of voltage is smaller in comparison to the hypothetical linear system, large energy conversion over a range of frequencies more than two times wider is obtained with the nonlinear behaviour. Furthermore, a similar behaviour occurs for cross-well oscillations around the first bending mode of stable state 2 which provides the proposed nonlinear harvesting concept with a much larger band of large energy conversion than a linear counterpart. As mentioned in section 3.2 only a small part of the cross-well oscillations around the first mode of state 2 could be investigated with the available equipment, which are shown as black crosses in Fig. 7. The resulting range for which cross-well oscillations are triggered is thus widened by an additional $2.5 \mathrm{~Hz}$ considering the dynamics of stable state 2, hence a total bandwidth of approximately $5 \mathrm{~Hz}$ of large power conversion is possible with the studied specimen. It is important to remark that this range is wider for lower frequencies than $17.5 \mathrm{~Hz}$, however adequate results could not be obtained due to the limits of the used electromechanical shaker.

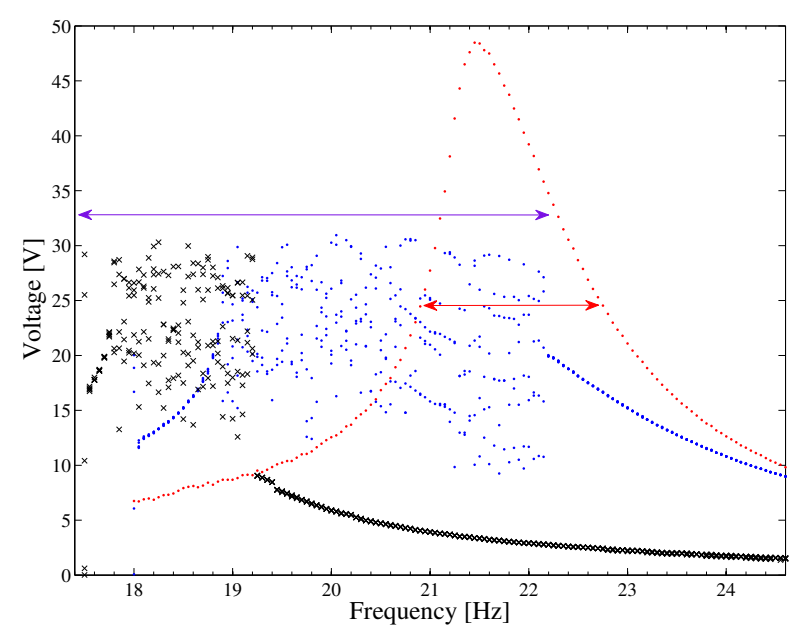

Figure 7. Frequency response diagram for the voltage amplitude against forcing frequency at a base acceleration level of $0.25 \mathrm{~g}$ load resistance of $27 \mathrm{k} \Omega$. Purple arrow shows the range of frequencies for which cross-well oscillations occur, The red arrow shows the linear range at $50 \%$ of peak voltage for the hypothetical linear system. 


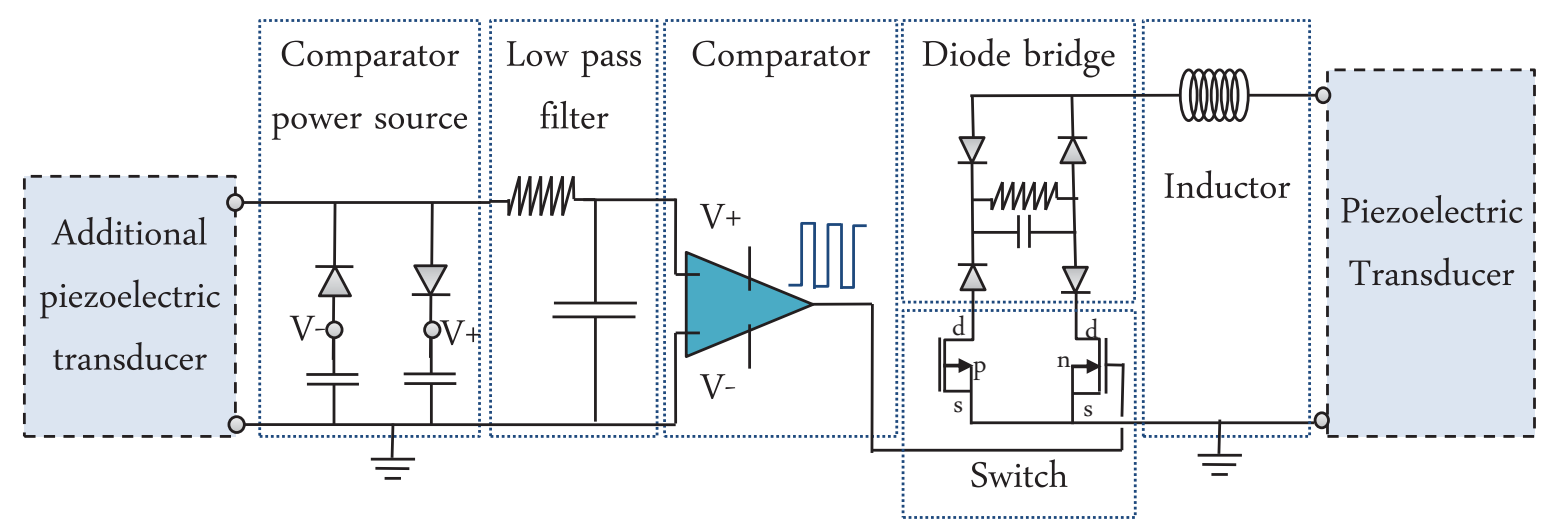

Figure 8. Synchronized Switch Harvesting on Inductor circuit used for broadband conversion.

\subsection{Synchronized Switching Harvesting}

Although the previous sections were focused on the analysis of the dynamic response of the electromechanical system, a stable DC signal is required as an output of the energy harvester in order to be effectively used, for instance, to charge a battery. The voltage produced by the piezoelectric elements can be simply rectified with a standard diode bridge, or more sophisticated techniques can be used in order to increase the power output. An effective technique is the Synchronized Switch Harvesting on Inductor (SSHI), which was developed by Guyomar et al. ${ }^{16}$ as an extension of the Synchronized Switch Damping on Inductor (SSDI) technique of Richard et al. ${ }^{17}$ The SSHI consists of a series of an inductor, a switch and a rectifier bridge connected in parallel with the piezoelectric element. The SSHI is based on the idea of cyclically switching the electrical boundary condition from open circuit to a specific electric impedance state in order to obtain a fast change in the voltage across the piezoelectric element. The switch should be synchronized with the dynamic response of the structure so that it occurs in correspondence of extrema of the structural displacement. When the piezoelectric element is in the open circuit state, the electric charges are accumulated on the electrodes of the piezoelectric element. Only when the piezoelectric element is connected to the circuit, an electric current flows through charging the reservoir capacitor of the rectifier bridge. The SSHI has an intrinsic broadband capability, since none of its components need to be tuned to a specific frequency. It is therefore well suited to be applied to the bi-stable structure studied in this work, which exhibits as a main feature the possibility to harvest energy in a broad frequency range. In this work, the SSHI technique is implemented by extending the autonomous architecture of the SSDI proposed by Delpero et al. ${ }^{18}$ In particular, the implementation of the SSHI technique requires an additional part of the circuit responsible for the detection of the minima and the maxima of the displacement and the generation of the control signal for the switch. These tasks are fulfilled by an additional piezoelectric transducer that provides the control signal and the required energy to the switch, as shown in Fig. 8. The signal generated by the additional piezoelectric element is treated in a low pass-filter and amplified by a comparator in order to generate a square signal that drives the switch. The small power required by the comparator is harvested by a diode bridge in two capacitors from the same additional piezoelectric transducer, so that the circuit is completely autonomous. Hence, one of the surface bonded transducers is used to power the SSHI circuit, while the other converts energy from the external vibration source.

The results for the rectified power obtained using the SSHI circuit to convert energy from the broadband oscillations typical of the cross-well dynamics exhibited by the bi-stable composited showed very good harvesting performance, as shown in Fig. 9. Large power conversion ranging between 30 up to $56 \mathrm{~mW}$ is obtained with the piezoelectric cantilevered bi-stable composite harvester equipped with a switching shunt circuit for the range of $5 \mathrm{~Hz}$ described in section 4.1. This results become even more remarkable when noticing that this were obtained with only one piezoelectric working as an energy transducer. This constitutes a major step forward in harvesting technologies when compared with other types of broadband concepts. ${ }^{5,19}$ 


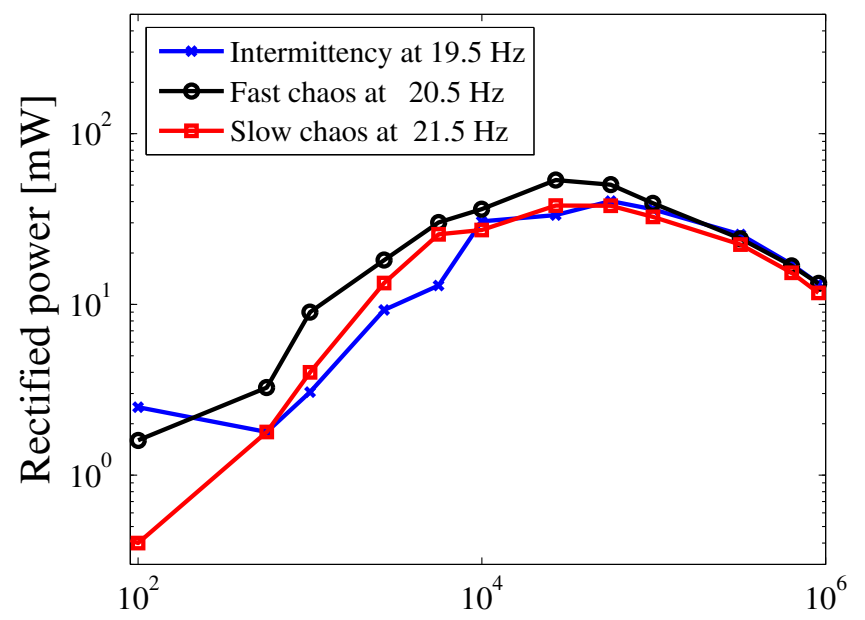

Figure 9. Experimental rectified power against load resistance of the SSH circuit for: intermittency oscillations at 19.5 $\mathrm{Hz}$, fast chaotic oscillations at $20.5 \mathrm{~Hz}$ and slow chaotic oscillations at $21.5 \mathrm{~Hz}$. Initial configuration state 1.

\section{CONCLUSIONS}

A novel concept for a broadband energy harvesting device using a piezoelectric bi-stable composite in a cantilevered configuration is presented. The new cantilevered configuration allows to exploit the large strains developed close to the clamped root, which results in large energy conversion while maintaning the broadband characteristics of previously presented harvesting concepts based on bi-stable composites. Thanks to the cantilevered configuration used in this paper, large deflections occur leading to snap-through being triggered at levels of base acceleration approximately 8 times lower than previously reported in literature. Furthermore, the reduction in the required level of base acceleration to obtain broadband energy conversion does not hinder the amount of converted energy. To further increase the effectiveness of the proposed concept, a synchronized shunting harvesting circuit well suited for conversion of broadband oscillations resulting from the cross-well dynamics of the bi-stable composites used is introduced showing excellent results in terms of very large levels of energy conversion. Rectified power values ranging between 30 to $56 \mathrm{~mW}$ are obtained with the proposed concept for a range of $5 \mathrm{~Hz}$. Such high levels of harvested power, converted with only one piezoelectric transducer of dimensions $50.8 \times 25.4 \times 0.152 \mathrm{~mm}$, over the obtained frequency range show the very high potential of the proposed concept for providing efficient and reliable autonomous energy supply.

\section{ACKNOWLEDGMENTS}

The authors would like to thank the support of the ETH Research Commission and the Marie Curie Actions Cofund Program; Dr. A. F. Arrieta is funded through an ETH Postdoctoral Fellowship.

\section{REFERENCES}

[1] S. Priya and D. J. Inman, editors. Energy Harvesting Technologies. Springer, 2009.

[2] C. Williams and R. Yates. Analysis of a micro-electric generator for microsystems. Sensors Actuators, $52: 811,1996$.

[3] H. A. Sodano, G. E. Simmers, R. Dereux, and D. J. Inman. Recharging batteries using energy harvested from thermal gradients. Journal of Intelligent Material Systems and Structures, 18:3-10, 2007.

[4] S. R. Anton and H. A. Sodano. A review of power harvesting using piezoelectric materials (20032006). Smart Materials and Structures, 16:R1-21, 2007.

[5] L. Gammaitoni, I. Neri, and H. Vocca. Nonlinear oscillators for vibration energy harvesting. Applied Physics Letters, 94:164102, 2009. 
[6] A. F. Arrieta, P. Hagedorn, A. Erturk, and D. J. Inman. A piezoelectric bi-stable plate for nonlinear broadband energy harvesting. Applied Physics Letters, 97:104102, 2010.

[7] M. W. Hyer. Some observations on the cured shapes of thin unsymmetric laminates. Journal of Composite Materials, 15:175-194, 1981.

[8] M. L. Dano and M. W. Hyer. Sma-induced snap-through of unsymmetric fiber-reinforced composite laminates. International Journal of Solids and Structures, 40:5949-5972, 2003.

[9] A. F. Arrieta, S. A. Neild, and D. J. Wagg. On the cross-well dynamics of a bi-stable composite plate. Journal of Sound and Vibration, 330:3424-3441, 2011.

[10] A. F. Arrieta, O. Bilgen, M. I. Friswell, and P. Hagedorn. Dynamic control for morphing of bi-stable composites. Journal of Intelligent Material Systems and Structures, 24:266-273, 2012.

[11] A. F. Arrieta, D. J. Wagg, and S. A. Neild. Dynamic snap-through for morphing of bi-stable composite plates. Journal of Intelligent Material Systems and Structures, 22:103-112, 2011.

[12] F. Mattioni. Thermally induced multi-stable composites for morphing aircraft applications. PhD thesis, University of Bristol, 2009.

[13] SLG Group: The carbon company, http://www.sglgroup.com. High-Performance Prepregs: Preimpregnated Products for Fiber-Reinforced Composites, 2011.

[14] A. F. Arrieta, A. Erturk, P. Hagedorn, and D. J. Inman. Electromechanical modelling and experiments of a bistable plate for nonlinear energy harvesting. In ASME 2010 Conference on Smart Structures, Adaptive Structures and Intelligent Systems (SMASIS2010), September 28 - October 1, 2010, Philadelphia, Pennsylvania, USA, 2010.

[15] A. F. Arrieta, S.A. Neild, and D.J. Wagg. Nonlinear dynamic response and modelling of a bi-stable composite plate for applications to adaptive structures. Nonlinear Dynamics, 58:259-272, 2009.

[16] D. Guyomar, A. Badel, E. Lefeuvre, and C. Richard. Toward energy harvesting using active materials and conversion improvement by nonlinear processing. IEEE Transactions of Ultrasonics, Ferroelectrics, and Frequency Control,, 52:584595, 2005.

[17] C. Richard, D. Guyomar, D. Audigier, and G. Ching. Semi-passive damping using continuous switching of a piezoelectric device. In Proceedings of the SPIE, 1998.

[18] T. Delpero, L. Di Lillo, A. Bergamini, and P. Ermanni. Energy harvesting module for the improvement of the damping performance of autonomous synchronized switching on inductance. Journal of Intelligent Material Systems and Structures, Published Online First, 2012.

[19] R. Ramlan, M. J. Brennan, B. R. Mace, and I. Kovacic. Potential benefits of a non-linear stiffness in an energy harvesting device. Nonlinear Dynamics, 59:545-558, 2010. 